\title{
DEGRADAÇÃO DE ÉSTERES DE FORBOL PRESENTES NA TORTA DE PINHÃO MANSO UTILIZANDO FUNGO LIPOLÍTICO
}

\author{
H. B. CARMINATI ${ }^{1}$, L. P. BORGES ${ }^{1}$, T. A. MONTARDI ${ }^{1}$, T. C. PIN $^{1}$, L. G. SANTOS ${ }^{1}$, P. W. \\ P. ANTUNES ${ }^{2}$, R. N. OSS ${ }^{2}$, M. O. D. MARTINS ${ }^{1}$ \\ ${ }^{1}$ Faculdades Integradas de Aracruz, Departamento de Engenharia Química \\ ${ }^{2}$ Universidade Federal do Espírito Santo, Departamento de Engenharia Ambiental \\ E-mail para contato: thaynara_coradini@hotmail.com
}

\begin{abstract}
RESUMO - A crescente demanda por energia impeliu a procura por novas fontes combustíveis, sendo o biodiesel um forte candidato. O Brasil possui uma variedade de oleaginosas com potencial para produção desse biocombustível, dentre elas o pinhão manso (Jatropha curcas L.). A extração do óleo dessa fonte gera uma torta rica em proteínas, carboidratos e lipídios, além de substâncias alergênicas e tóxicas que inviabilizam o seu uso, das quais, se incluem os ésteres de forbol. Diante desse entrave, o presente trabalho visa a degradação desses ésteres presentes no resíduo, através da inoculação do meio com fungos do gênero Aspergillus coletados de fontes distintas e capazes de crescer na torta. Os níveis dos ésteres de forbol presentes na torta foram verificados por Cromatografia Líquida de Alta Eficiência (CLAE) e teste ecotoxicológico com a bactéria Vibrio Fischeri. Pode-se constatar, pelas análises, uma diminuição dos níveis de ésteres de forbol em $55 \%$, validando a eficácia do fungo na degradação dessas substâncias tóxicas.
\end{abstract}

\section{INTRODUÇÃO}

Um constante desafio para a sociedade é suprir sua demanda energética, fator que permitiu um grande enfoque nos estudos de fontes renováveis de energia. O biodiesel é considerado como uma importante fonte de energia renovável e constituindo uma alternativa potencial para a redução da emissão de gases de efeito estufa no setor de transportes (BASHA; GOPAL; JEBARAJ, 2010).

Esse combustível renovável pode ser obtido a partir de óleos vegetais, dos qual o Brasil possui uma grande variedade de fontes oleaginosas. Entre elas estão: soja, dendê (palma), mamona, girassol, canola e pinhão manso, as quais possibilitam a extração de óleos vegetais para a produção de biodiesel em larga escala, (MELO et al, 2010).

A expansão do cultivo de oleaginosas para a produção de biodiesel tem gerado um crescimento e diversificação de tortas e farelos que devem ser aproveitados de forma a melhorar a sustentabilidade da cadeia produtiva (FARIA-MACHADO et al., 2011). Um exemplar de 


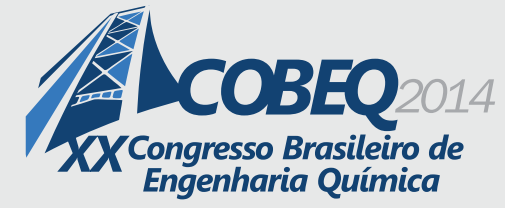

19 a 22 de outubro de 2014

Florianópolis/SC

oleaginosa é o pinhão manso, (Jatropha curcas L.) uma espécie perene e monoica, pertencente à família das Euforbiáceas (HELLER, 1996 apud BELTRÃO, 2005).

Os resíduos são produzidos em larga escada, cuja composição inclui carboidratos, glicídios, proteínas e lipídeos (SANTOS; SANTOS; MACEDO, 2009). Entretanto, uma peculiaridade do resíduo de pinhão manso é a presença de algumas substâncias tóxicas e alergênicas, que descaracterizam do mesmo (TAMALAMPUDI et al., 2008).

Os efeitos tóxicos e alergênicos do pinhão manso relacionam-se com a presença de substâncias como: curcina, ésteres de forbol e de uma proteína com potencial alergênico semelhante à albumina 2S da mamona (MACIEL; MACHADO, 2007; MARTINEZ-HERRERA et al., 2006), entre os quais, os mais nocivos são os ésteres de forbol (diterpenos). Tal fato se deve à gravidade desta toxina, que pode agir em organismos de duas formas: uma aguda (resposta inflamatória intensa) e outra crônica (indutores da formação de tumores) (MAKKAR et al., 1997).

Dentre as metodologias de detoxificação que visam a eliminação de ésteres de forbol do pinhão manso, existem os tratamentos químicos e microbianos. Os tratamentos químicos são mais agressivos ao meio ambiente, uma vez que uma grande quantidade de metanol é utilizada (JOSHI; PRIYANKA; KHARE, 2011). O processo enzimático, por sua vez, envolve a utilização de microorganismos capazes de produzir enzimas lipolíticas que atuam na degradação dos ésteres de forbol (MACIEL et al., 2010).

Frente a um aumento significativo da quantidade de torta gerada (aproximadamente 58,5 mil toneladas/ano) (RIBEIRO et al., 2010) como subproduto da cadeia produtiva de biodiesel, é oportuna a aplicação de uma técnica que viabilize o aproveitamento desse resíduo. A utilização de enzimas, em especial as lipases, surge como uma alternativa promissora, haja vista que tais enzimas atuam na degradação dos ésteres de forbol com baixa geração de novos resíduos, e ainda com possibilidade de reutilização das mesmas no processo (PORTELA, 2011; MACIEL et al., 2010). Nesse contexto, esse trabalho visa estudar uma forma eficiente para a detoxificação dos ésteres de forbol.

\section{MATERIAIS E MÉTODOS}

A torta do pinhão manso foi disponibilizada por uma empresa do ramo energético do norte do Espírito Santo. A mesma passou por uma caracterização físico-química visando elencar possíveis parâmetros relevantes, tais como umidade, distribuição granulométrica e teores de lipídeos, carboidratos e proteínas. A caracterização garantiu também a reprodutibilidade dos resultados experimentais. 


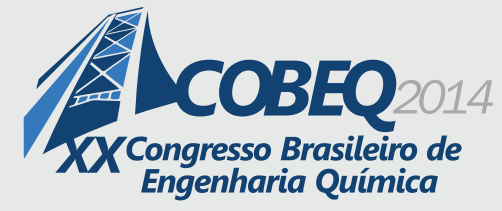

\subsection{Estudo de Detoxicação do Resíduo de Pinhão Manso}

A detoxificação do pinhão manso é realizada por processo enzimático, e vem sendo pesquisada para fornecer uma alternativa a este tratamento. Esse método consiste em utilizar microrganismos, que em diferentes condições de cultura, são capazes de produzir enzimas lipolíticas, as quais agirão na degradação dos ésteres (MACIEL et al., 2010). Tal método é considerado ambientalmente correto e apresenta diversas vantagens (PORTELA, 2011).

A detoxificação foi realizada com o preparo do inóculo utilizando $5 \mathrm{~g} / \mathrm{L}$ de peptona, $5 \mathrm{~g} / \mathrm{L}$ de extrato de leveduras e $0,5 \mathrm{~g} / \mathrm{L}$ de $\mathrm{NaCl}$, e posteriormente este meio foi esterilizado em autoclave (marca Quimis, modelo Q290-12). Esterilizado, o meio teve seu pH corrigido para 7,0 utilizando $\mathrm{NaOH}$, para então receber a cultura de fungos. Por fim o preparo foi encaminhado para um processo de agitadação por 24 horas em um shaker orbital de dupla ação (marca Marconi, modelo MA570) à temperatura de $30^{\circ} \mathrm{C}$.

A cultura de fungos utilizada foi Aspergillus, oriunda do fruto do tomate. A obtenção das colônias foi realizada através da coleta de esporos de cascas do fruto, seguida de isolamento e cultivo em meios adequados. Um estudo preliminar permitiu determinar uma atividade lipolítica média de $12,24 \mathrm{pNP} / \mathrm{min}$ para o fungo.

Para preparar o substrato adicionou-se $5 \mathrm{~g}$ da torta de pinhão manso e $5 \mathrm{~mL}$ de água destilada esterilizada (para umidificação da torta) em um erlenmeyer de $250 \mathrm{~mL}$. Posteriormente, o erlenmeyer foi esterilizado em autoclave (marca Quimis, modelo Q290-12). Em seguida, fez-se a inoculação, adicionando-se ao erlenmeyer $2 \mathrm{~mL}$ da suspensão de microorganismos, cuja concentração foi de aproximadamente $10^{7}$ células $/ \mathrm{mL}$. Para a contagem das células foi utilizada a câmara de Neubauer. A suspensão preparada foi observada durante 7 dias, ocorrendo assim a possível degradação dos ésteres de forbol.

As condições experimentais foram adotadas conforme aquelas utilizadas nos trabalhos de Maciel et al. (2010), Saetae e Suntornsuk (2010), Portela (2011) entre outros. Alguns dos mesmos sugerem a utilização de diferentes culturas de fungos lipolíticos, por exemplo, aqueles oriundos de resíduos oleosos. Contudo, essas e outras alternativas propostas não foram adotadas nesse trabalho, tendo em vista que não apresentaram resultados tão satisfatórios.

Para a análise da porcentagem de degradação dos ésteres de forbol, fez-se a extração destes, seguindo o método Saetae e Suntornsuk (2010), que consiste na adição de $20 \mathrm{~mL}$ de metanol em $2 \mathrm{~g}$ da torta de pinhão manso. A mistura foi agitada a $200 \mathrm{rpm}$ durante 5 minutos em um agitador shaker orbital de dupla ação (marca Marconi, modelo MA570). Em seguida foi realizada uma filtração a vácuo e o filtrado recolhido. O processo de extração foi repetido 4 vezes. As frações de extratos foram agrupadas e evaporadas em um evaporador rotativo (marca IKA, modelo HB $05.06 \mathrm{CN}$ ) a $65{ }^{\circ} \mathrm{C}$ para obtenção de frações concentradas de ésteres de forbol. A extração dos ésteres presentes na torta de pinhão manso foi realizada antes e depois da inoculação dos fungos, para a avaliação da degradação destes. 


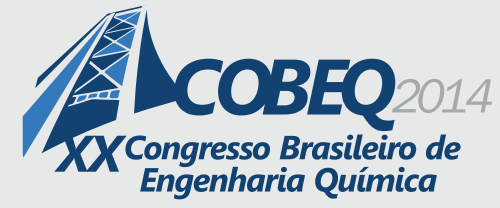

\subsection{Análise da Toxicidade do Sólido Fermentado}

A torta inoculada foi diluída com água destilada na proporção de 1:10, a mesma foi agitada em um shaker orbital de dupla ação (marca Marconi, modelo MA570) a $150 \mathrm{rpm}$, por 24 horas e a uma temperatura de $25^{\circ} \mathrm{C}$ e posteriormente, foi centrifugada (marca Quimis, modelo Q222B2). Em seguida, foi realizada outra diluição na mesma proporção, sendo que o limite de turbidez da amostra é de 50 NTUs. O pH da amostra foi corrigido para um intervalo entre 6,0 e 8,5.

Posteriormente, foram adicionados a cada um dos tubos de leitura do Ecotox (marca Microtox Acute Toxicity, modelo M500) uma alíquota de $0,5 \mathrm{~mL}$ da amostra e uma alíquota de $0,5 \mathrm{~mL}$ de bactérias Vibrio fischeri (utilizada como indicador), ficando o sistema com $50 \%$ da amostra e $50 \%$ com bactérias em seu meio. Esse sistema permaneceu por um período de tempo nos tubos para aclimatização. Todo esse procedimento foi realizado em termobloco com temperatura estabilizada a $15^{\circ} \mathrm{C}$. A leitura da luminescência da amostra foi realizada nos tempos de 0,5 e 15 minutos.

O mesmo procedimento foi realizado para a torta sem inoculação para fins comparativos.

\subsection{Análise da Degradação dos Ésteres de Forbol Presentes na Torta de Pinhão Manso}

Os ésteres de forbol extraídos da amostra foram analisados utilizando cromatografia líquida de alta performance (HPLC). Sendo o sistema de cromatografia da série Shimadzu CBM-20A, equipado com um desgaseificador de solventes DGU-20AS, uma bomba quaternária de gradiente LC-20AT, um injetor automático de amostras SIL-20AHT e um detector de arranjo de diodos SPD-M20A. A coluna cromatográfica utilizada neste estudo foi a coluna analítica KinetexTM C18 (100 x 2,1 mm, 2,6 $\mu \mathrm{m}, 100 \mathrm{~nm})$. O comprimento de onda de detecção foi de $280 \mathrm{~nm}$. Para as análises foram injetadas $50 \mu \mathrm{L}$ da amostra e a taxa de fluxo da fase móvel foi de $0,400 \mathrm{~mL} / \mathrm{min}$ e teve como padrão externo forbol-12-miristato-13-acetato (PMA) (marca Sigma-Aldrich). A temperatura da coluna foi mantida constante em $30{ }^{\circ} \mathrm{C}$, utilizando um módulo aquecedor de coluna da Shimadzu.

As análises cromatográficas foram realizadas em gradiente utilizando água e acetonitrila como fase móvel A e B, respectivamente. O perfil do gradiente iniciou-se com $40 \%$ da fase móvel B. Entre 0 e 15 minutos aplicou-se um gradiente linear de acetonitrila, variando a porcentagem da fase móvel B de 40 a $75 \%$. Entre 15 e 20 minutos, o gradiente linear variou de 75 a $100 \%$. A concentração de $100 \%$ de acetonitrila foi mantida até 25 minutos. Entre 25 e 26 minutos, o gradiente retornou à condição inicial e manteve-se isocrático até 36 minutos. Todas as soluções preparadas para compor as fases móveis foram previamente filtradas e mantidas por 15 minutos em banho de ultrason (Limpsonic $®$, Brasil) antes de serem utilizadas. 


\section{RESULTADOS}

\subsection{Determinação da Toxicidade por Ecotox}

$\mathrm{O}$ Ecotox determina a concentração efetiva média $\left(\mathrm{CE}_{50}\right)$ das amostras em diferentes concentrações. Sendo que para o primeiro teste é realizada uma diluição obtendo uma concentração de $45 \%$. Porém, se o limite da escala do equipamento for atingido, este realiza novas diluições, a fim de garantir que os valores de $\mathrm{CE}_{50}$ estejam dentro dos limites.

Nas amostras em questão, o teste foi realizado na concentração de $45 \%$, obtendo valores satisfatórios e com o erro minimizado. De acordo com Magalhães e Ferrão Filho (2008), $\mathrm{CE}_{50}$ é a concentração do agente tóxico que causa mortalidade/imobilidade a 50\% dos organismos-teste depois de um determinado tempo de exposição. Assim, os valores obtidos para as amostras em testes podem ser observados na Tabela 2 . Sendo que foram realizadas duas análises, a primeira na torta sem inóculo e outra na torta após a inoculação com o fungo Aspergillus.

Tabela 2 - Valores de concentração efetiva média $\left(\mathrm{CE}_{50}\right)$

\begin{tabular}{cc}
\hline Amostra & $\mathrm{CE}_{50}(\%)$ \\
\hline Torta & 14,51 \\
Torta + inóculo (fungo Aspergillus) & 70,46 \\
\hline
\end{tabular}

De acordo com Costa et al. (2008), o valor numérico de toxicidade aguda, expresso como $\mathrm{CE}_{50}$, exprime uma relação inversa à toxicidade, ou seja, maiores valores numéricos indicam menores toxicidades. Sendo assim, o $\mathrm{CE}_{50}$ passou de $14,51 \%$ para $70,46 \%$ após a inoculação, indicando uma diminuição na toxicidade da torta.

\subsection{Detecção de Ésteres de Forbol Utilizando HPLC}

A análise dos ésteres de forbol extraídos foi realizada por sobreposição dos perfis cromatográficos obtidos. O perfil da amostra de PMA diluído em metanol (10 $\mu \mathrm{g} / \mathrm{L})$ foi utilizado com padrão externo. O PMA é comumente utilizado como padrão externo, pois apresenta um tempo de retenção próximo aos ésteres de forbol presentes em J. curcas (JOSHI; PRIYANKA; KHARE, 2011).

A eluição do padrão de PMA apresentou um tempo de retenção de 24,7 minutos. No perfil cromatográfico do controle (amostra sem inóculo), próximos a esse tempo de retenção, foram identificados quatro outros picos, eluídos respectivamente, em 21,9 minutos; 29,5 minutos; 30,1 minutos e 31,0 minutos (Gráfico 2), sendo estes os ésteres de forbol (EF). 


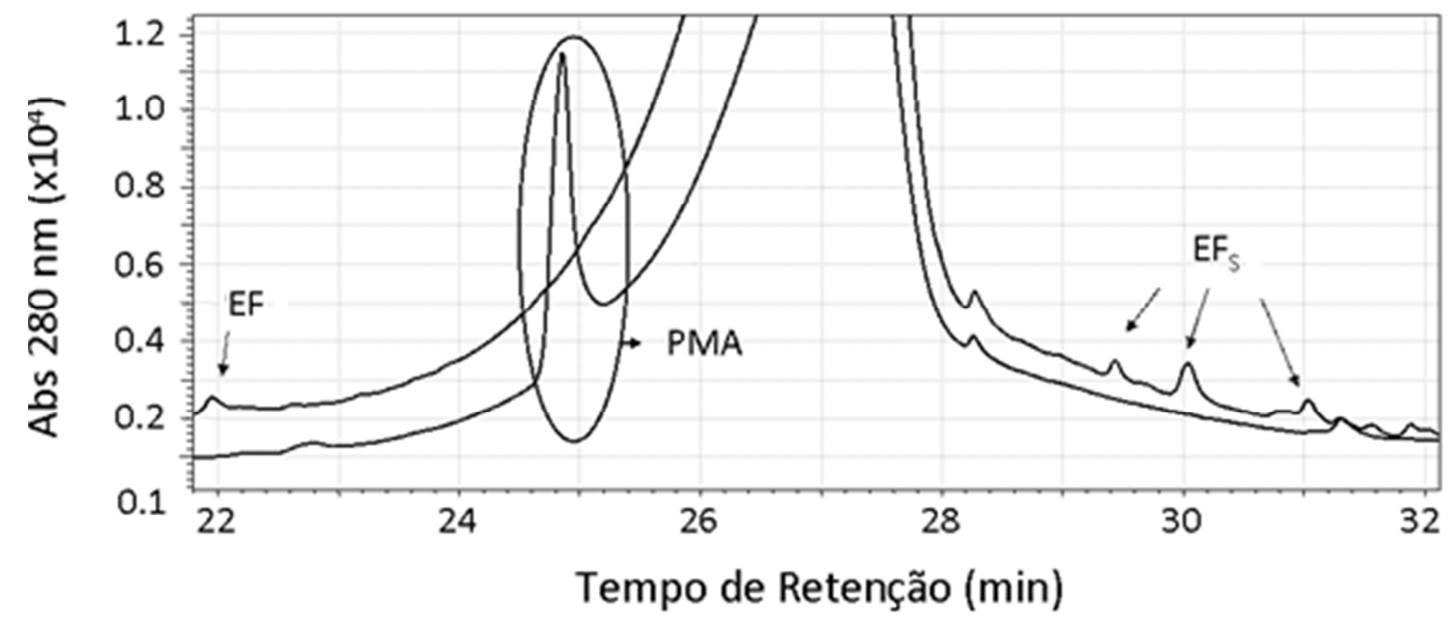

Gráfico 2 - Perfil cromatográfico do padrão externo e do controle.

Segundo Joshi et al. (2011), a Jatropha sp. apresenta de 4 a 6 ésteres de forbol e seus derivados. Estudos demonstram a existência de até mesmo mais de 6 ésteres de forbol em $J$. curcas, dos quais, o PMA é sugerido como sendo o principal deles (MAKKAR et al., 1997; HAAS et al., 2000), porém não foi observada a presença deste nas amostras avaliadas.

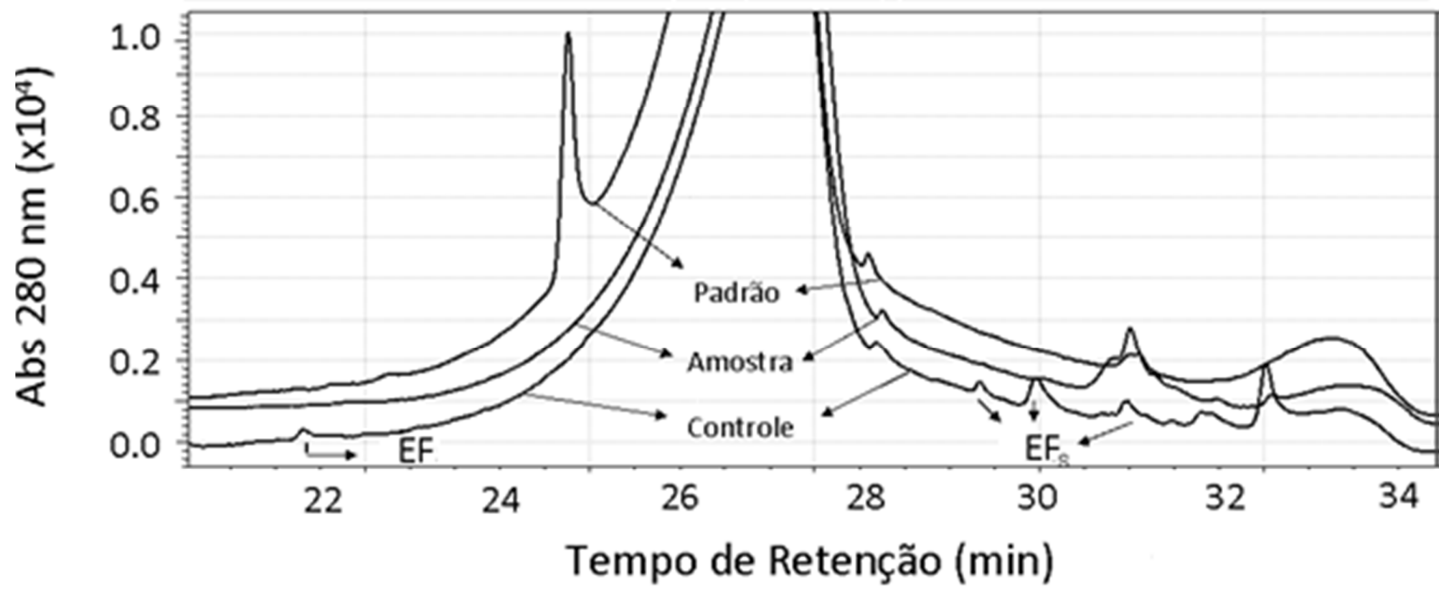

Gráfico 3 - Perfil cromatográfico do padrão externo, do controle e da amostra com o inóculo.

Segundo Martinez-Herrera et al. (2006), os tipos de ésteres de forbol e suas características químicas estão relacionados com a variedade da planta e, principalmente, com as condições de clima e solo para o seu crescimento. Estudos realizados com três diferentes variedades tropicais de J. curcas da Malásia, Indonésia e Índia, demonstraram 2, 5 e 4 picos de ésteres de forbol, respectivamente (AHMED; SALIMON, 2009). Devido a grande variedade nas características e 
na quantidade de ésteres de forbol encontrados nos diferentes estudos, foram utilizados os 4 picos eluídos próximos de PMA (Gráfico 2) para realização dos estudos de degradação.

O perfil cromatográfico do controle (sem o inóculo Aspergillus) comparado ao da amostra fermentada (com o inóculo Aspergillus) demonstrou ausência de três dos quatros picos de $\mathrm{EF}_{\mathrm{S}}$ (Gráfico 3). A ausência dos picos eluídos em 21,9 minutos; 29,5 minutos e 30,1 minutos é um possível indicativo que a fermentação utilizando o fungo Aspergillus possui um potencial de degradação de ésteres de forbol, podendo este ser utilizado como uma alternativa aos tratamentos químicos.

\section{CONCLUSÕES}

A realização do presente trabalho permitiu concluir que a utilização de fungo lipolítico visando à degradação de ésteres de forbol presentes na torta de pinhão manso, é uma alternativa viável na detoxificação. Assim, o fungo Aspergillus apresentou bom potencial para degradação dos ésteres de forbol.

Com a análise por Ecotox e por HPLC, foi observada a diminuição dos níveis de ésteres de forbol presentes na torta de pinhão manso, confirmando o potencial lipolítico do fungo Aspergillus em degradar essa substância tóxica.

\section{REFERÊNCIAS}

AHMED, W. L.; SALIMON, J. Phorbol ester as toxic constituents of tropical Jatropha curcas seed oil. Eur. J. Sci. Res., v. 31, p. 429-436, 2009.

BASHA, S. A; GOPAL, K. R; JEBARAJ, S. A review on biodiesel production, combustion, emissions and performance, p. 3155- 3161, 2010.

BELTRÃO, N. E. M. Agronegócio das oleaginosas no Brasil. Inf. Agropec., 26:44-78, 2005.

BROOKER, D. B.; BAKKER-ARKEMA, F. W.; HALL, C. W. Drying and storage of grains and oilseeds. Westport: AVI, p. 450, 1992.

COSTA, C. R.; OlIVI, P.; BOTTA, C. M. R.; ESPINDOLA, E. L. G. A toxicidade em ambientes aquáticos: discussão e métodos de avaliação. Química Nova, v. 31, n. 7, 2008.

FARIA-MACHADO, A. F.; SOUZA, M. L. M.; CASTRO, I. M.; BIZZO, H. R.; MENDONÇA, S.; ANTONIASSI, R. Ésteres de forbol em tortas de pinhão manso. II Congresso Brasileiro de Pesquisas de Pinhão-Manso. Brasília, DF, 2011.

HAAS, W.; MITTELBACH, M. Detoxification experiments with the seed oil from Jatropha curcas L. Ind. Crop. Prod. v. 12, p. 111-118, 2000. 


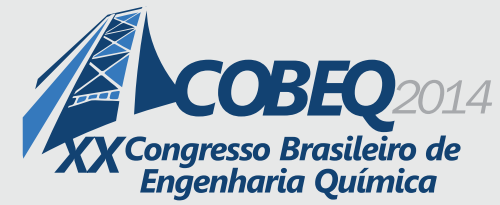

JOSHI, C.; PRIYANKA, M.; KHARE, P. M. Degradation of phorbol esters by Pseudomonas aeruginosa PseA during solid-state fermentation of deoiled Jatropha curcas seed cake. India. 2011.

MACIEL, F. M.; MACHADO, O. L. T. Avaliação do potencial alergênico de sementes de Jatropha Curcas L., pinhão-manso. In: II Congresso da Rede Brasileira de Tecnologia de Biodiesel, 2., 2007, Brasília, DF. Livro de resumos... Brasília, DF: MCT: ABIPTI, 2007.

MACIEL, V. F. A.; POLETTO, C. M.; MENDONÇA, S.; GONÇALVES, S. B. Seleção de linhagens para degradação do forbol de pinhão manso. Embrapa Agroenergia. Brasília, 2010.

MAGALHÃES, D. P.; FERRÃO FILHO, A. S. A ecotoxicologia como ferramenta do biomonitoramento de ecossistemas aquáticos. Instituto Oswaldo Cruz, Rio de Janeiro, 2008.

MAKKAR, H. P. S.; BECKER, K.; SPORE, F.; WINK, M. Studies on nutritive potential and toxic constituents of different provenances of Jatropha curcas. J. of Agriculture and Food Chem., Easton, n. 45, p. 3152-3157, 1997.

MARTINEZ-HERRERA， J.; SIDDHURAJU， P.; FRANCIS， G.; DAVILA-ORTIZ， G.; BECKER, K. Chemical composition, toxic/antimetabolic constituents, and effects of different treatments on their levels, in four provenances of Jatropha curcas L. From Mexico. Food Chemistry, London, v. 96, p. 80-89, 2006.

MELO, J. C.; BRANDER JUNIOR., W.; CAMPOS, R. J. A.; PACHECO, J. G. A.; SCHULER, A. R. P.; STRAGEVITCH, L. Avaliação Preliminar do Potencial do Pinhão Manso para a Produção de Biodiesel. Recife, PE, 2010.

PORTELA, F. M. Efeito da catálise ácida e alcalina na produção e propriedades físico-químicas do biodiesel metílico de pinhão-manso. 2011. Dissertação (Mestrado) - Instituto de Química, Universidade Federal de Uberlândia, Minas Gerais, 2011.

RIBEIRO, J. A.; SCHWERZ JÚNIOR, P.; SOUSA, P. M.; MENDONÇA, S.; Desenvolvimento de processo semiautomatizado para extração e quantificação de ésteres de forbol em torta de pinhão-manso (Jatropha curcas L.) In: $7^{\circ}$ Congresso Brasileiro de Plantas Oleaginosas, Óleos, Gorduras e Biodiesel, p. 1, Belo horizonte, Minas Gerais, 2010.

SAETAE, D., SUNTORNSUK, W. Antifungal activities of ethanolic extract from Jatropha curcas seed cake. J. Microbiol Biotechnol. v. 20, p. 319-324, 2010.

SANTOS, A. S.; SANTOS, R. S.; MACEDO, A. L. Avaliação do aproveitamento da torta de pinhão-manso (Jatropha curcas L.) para a produção de bioetanol. Brasília, DF. 2009.

SOUZA, A. D. V; FÁVARO, S. P.; ÍTAVO, L. C. V. ROSCOER, R. Caracterização química de sementes e torta de pinhão manso, nabo forrageiro e crambe. Brasília. 2009.

TAMALAMPUDI, S.; TALUKDER, M. R.; HAMAD, S.; NUMATAB, T.; KONDO, A; FUKUDA, H. Enzymathic production of biodiesel from Jatropha oil - A comparative study of immobilized-whole cell and commercial lipases as a biocatalyst. J. Biochemical Eng., v. 39(1), p.185-189, 2008. 